\title{
Influencia del Acabado Superficial en el Digitalizado con Sensores de Triangulación por Láser
}

\author{
Eduardo Cuesta, Pedro Fernández, Braulio J. Álvarez y David Blanco \\ Universidad de Oviedo, Departamento de Construcción e Ingeniería de Fabricación, Campus de \\ Gijón (Viesques), Edificio Oeste nº 5, 33203 Gijón (Asturias)-España (email: ecuesta@uniovi.es)
}

\begin{abstract}
Resumen
El presente trabajo trata sobre la medida de la influencia que los distintos acabados superficiales tienen sobre la precisión y calidad del digitalizado utilizando sensores láser de triangulación. Entre el gran número de parámetros que influyen en la calidad del digitalizado, se analiza la influencia de la rugosidad. Para ello se ha establecido un procedimiento que permite restringir la influencia de otras variables y que, al mismo tiempo, proporciona una excelente repetibilidad y reproducibilidad. Este procedimiento se ha aplicado a distintos procesos de fabricación que dan lugar a muy distintos acabados superficiales. De esta forma se han establecido ciertas correlaciones cuantificando, además de la influencia de la rugosidad en la nube de puntos, la forma espacial que adopta su dispersión y la intensidad optima para cada proceso.
\end{abstract}

Palabras clave: digitalizado láser, acabado superficial, sensores láser, rugosidad

\section{Influence of Surface Finishing on the Scanning with Laser Triangulation Sensors}

\begin{abstract}
The present work deals with the measurement of the influence that surface finishing has on the accuracy and quality of the scanning performed using laser triangulation sensors. Amongst the great number of parameters that influence the scanning quality, the effect of roughness is analysed. To achieve this, a procedure for constraining the variables while providing an excellent repeatability and reproducibility was proposed. This procedure has been applied to several manufacturing processes that produce parts with very different quality of surface finishing. As a result, certain correlations have been extracted that quantify the influence of the roughness on the quality of the cloud-point, the spatial distribution of the cloud-point dispersion and the optimal laser intensity for each manufacturing process.
\end{abstract}

Keywords: laser scanning, surface finishing, laser sensors, roughness 


\section{INTRODUCCIÓN}

Los sensores por triangulación láser (LTS, Laser Triangulation Sensor) obtienen las coordenadas de miles de puntos por segundo utilizando un haz láser que oscila, construyendo una cortina (2D) que incide sobre un objeto o pieza (láser de cortina o de barrido). La alta velocidad de captura, unido a la ausencia de contacto, permite múltiples propósitos, desde ingeniería inversa (Lai et al., 2001; Son et al., 2002) hasta reconstrucciones infográficas (arte, urbanismo, cine, etc.) pasando por la inspección y verificación continua en una línea de producción (Li et al., 2002; Cuesta et al., 2005).

En la actualidad esta tecnología de digitalizado es aplicada en industrias con productos y requerimientos de fabricación de calidad aceptable con plazos cortos de entrega. Por otra parte, también es aplicada en industrias con piezas o productos de elevado tamaño y/o de geometrías complicadas difíciles de inspeccionar por métodos tradicionales. Estas últimas se aprovechan de la portabilidad que ofrecen los sensores montados sobre brazos de medición o sobre manipuladores implementados a medida de las necesidades de cada usuario. En general, son muchas las empresas que se plantean migrar hacia la inspección sin contacto, bien con sensores láser sobre brazos de medición, sobre Máquinas de Medir por Coordenadas (MMC), con láser trackers volumétricos, etc.

A pesar de las ventajas citadas, todavía existen barreras difíciles de superar. El coste de estos equipos todavía es muy elevado, y el conocimiento de los errores (en términos de incertidumbres) y precisiones que se pueden alcanzar es en gran medida desconocido (Feng et al., 2001; Rico et al., 2005a; Santolaria et al., 2005). Esto ocurre incluso en el caso de sensores ópticos montados sobre MMC (Keferstein y Zust, 2004) como es el caso del presente trabajo. Una de las razones fundamentales de esta falta de caracterización se encuentra en la enorme variedad de factores que pueden afectar a la cantidad y calidad de los puntos capturados. Además, y como se verá mas adelante, para obtener una nube de puntos que se ajuste óptimamente a la superficie real deben tenerse en cuenta también las trayectorias de digitalizado, es decir, el ángulo de incidencia del haz sobre la superficie (Curless y Levoy, 1995; Xi y Shu, 1999; Rico et al., 2005b).

En este trabajo se analiza la influencia de las variables más significativas del proceso sobre el acabado superficial de la pieza digitalizada, focalizando el estudio sobre la calidad y precisión del digitalizado.

\section{FUNDAMENTOS DE LOS SENSORES LÁSER POR TRIANGULACION. PARÁMETROS DE INFLUENCIA}

La mayoría de los sensores láser por triangulación para aplicaciones de ingeniería inversa presentan el mismo diseño constructivo (Fig. 1). Estos dispositivos obtienen por triangulación las coordenadas de los puntos de la superficie de la pieza mediante la imagen de los mismos que es captada por un

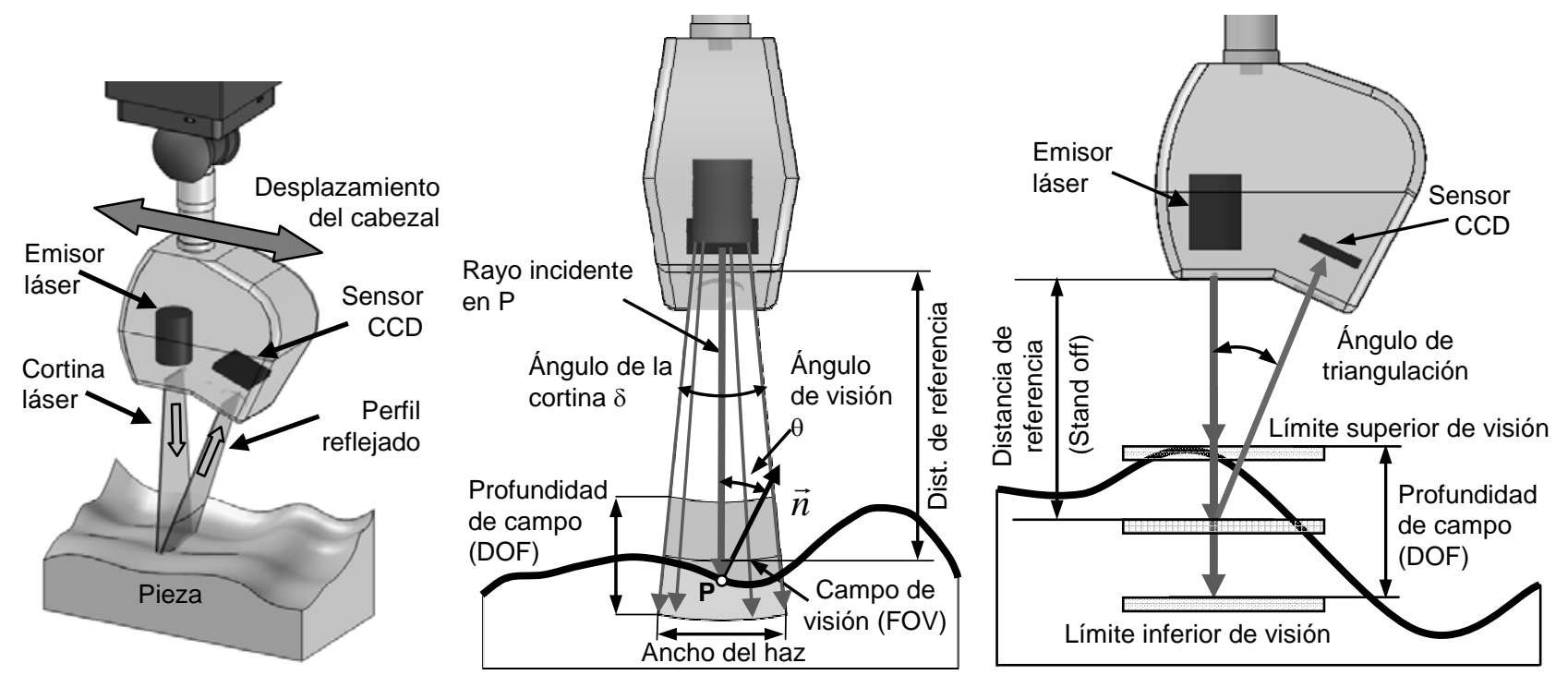

Fig. 1: Detalle de funcionamiento y parámetros geométricos del sensor láser. 

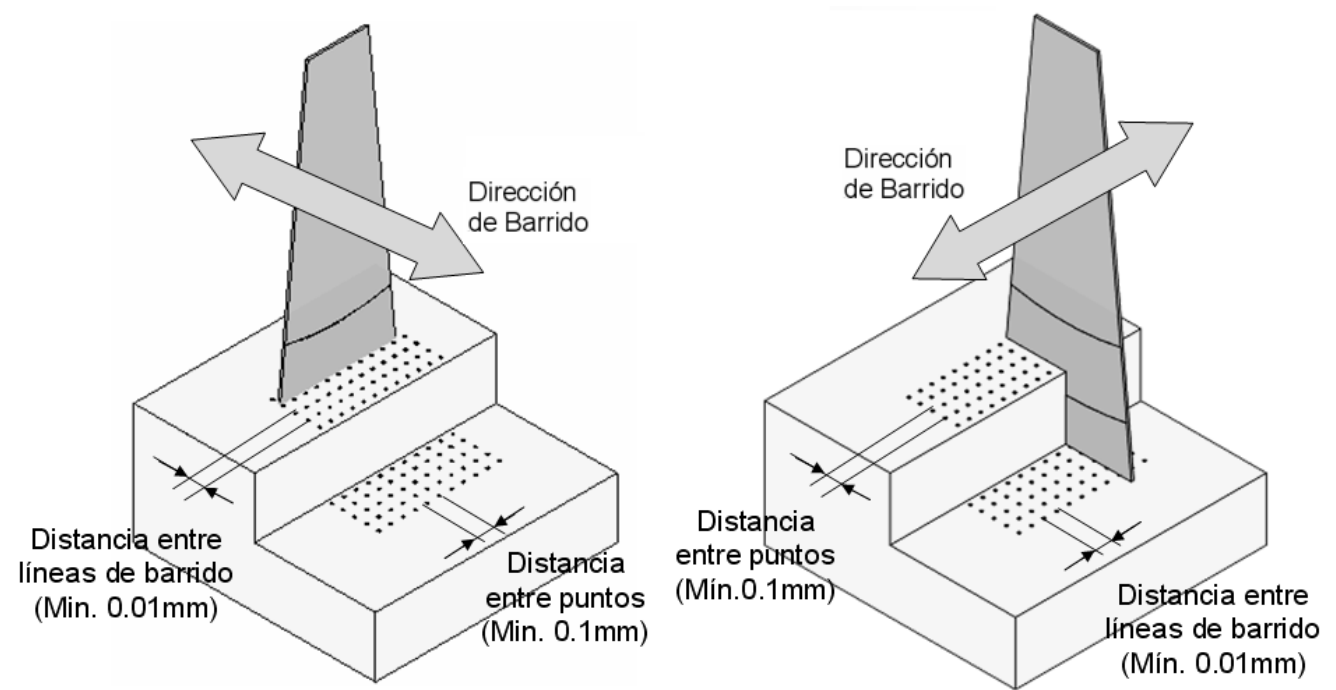

Fig. 2: Resolución máxima (distancia entre puntos) de las líneas de la cortina láser.

dispositivo fotodetector tipo CCD. El haz láser incidente se transforma en una cortina láser por medio de ópticas especiales o por un espejo rotativo acoplado al emisor. La Fig.1 muestra los parámetros geométricos y constructivos que definen el campo de actuación y la precisión de este sistema. El sensor utilizado en el presente trabajo es un sensor orientado a la detección de luz difusa (no especular) producida en la superficie del objeto. Se trata del modelo LC-50 de Metris@ montado sobre una MMC (Global Image $\odot$ de Brown\&Sharpe) y que permite velocidades de captura de 19.200 puntos por segundo.

La geometría constructiva del sistema permite definir una serie de parámetros geométricos no modificables, como son la distancia de referencia o de focalización (stand-off), el ángulo de triangulación o ángulo entre el haz incidente y el reflejado, y la profundidad de campo (Depth of Field, DOF). También debe considerarse el campo de visión (Field of view, FOV), que es la región dentro de la cual el sensor es capaz de recoger puntos sobre la superficie digitalizada. En el sensor utilizado, el campo de visión está definido por la profundidad de campo y el ancho del haz (aprox. $50 \times 50 \mathrm{~mm})$.

Por otro lado, existe un conjunto de parámetros tecnológicos configurables y que definen la experimentación del presente trabajo. Estos son: la intensidad del láser, la distancia entre los puntos de una misma línea de barrido y la distancia entre líneas de barrido (Fig. 2). La distancia entre puntos y líneas de barrido están relacionadas con la velocidad de adquisición de datos. La resolución máxima en este sensor coincide con el diámetro del haz láser más pequeño que puede distinguir el CCD, que es de $10 \mu \mathrm{m}$ en el sentido de avance del sensor y de $100 \mu \mathrm{m}$ entre puntos dentro de la línea de cortina láser. En cuanto a la intensidad del haz láser, puede ajustarse de 0 hasta 63, aprox. de 0 a $1 \mathrm{~mW}$. En otros sensores de triangulación, este parámetro es substituido por el tiempo de exposición. En ambos casos su variación produce un fuerte efecto sobre la calidad del digitalizado.

Para analizar la influencia de los parámetros geométricos se realizaron varios ensayos aislando cada uno de ellos. Por ejemplo, se ha comprobado que los valores óptimos de captura de puntos se producen cuando la superficie a digitalizar se encuentra entre la perpendicular al rayo incidente y la perpendicular al sensor CCD (dentro del ángulo de triangulación) y en la mitad de la profundidad de campo, es decir, a la distancia de referencia. Lejos de este punto perdían precisión aún teniendo en cuenta que existe una compensación por software de esas desviaciones. A esta misma conclusión han Ilegado otros investigadores (Keferstein y Marxer, 1998; Feng et al., 2001; Santolaria et al., 2005).

Otro grupo de ensayos se ha destinado a cuantificar la influencia de la iluminación externa en la calidad del digitalizado. No solo variando el tipo de luz (distintas longitudes de onda), sino también variando el ángulo de incidencia de la luz externa y su intensidad o cercanía al sensor. Las mejores precisiones, en cuanto a dispersión y repetibilidad de los puntos se han obtenido usando luz 
monocromática (lámpara de sodio) con una longitud de onda alejada de la del láser $(\lambda=635 \div 650$ nm), o mejor aún, en ausencia de luz.

\section{PROCEDIMIENTO PARA EVALUAR LA INFLUENCIA DEL ACABADO SUPERFICIAL}

Con objeto de evaluar exclusivamente la influencia que tienen los distintos acabados superficiales sobre el digitalizado láser fue necesario diseñar y evaluar un procedimiento que aislara los parámetros a estudiar, evitando en lo posible la influencia de otras magnitudes o parámetros que desvirtuarían los resultados (luz externa, geometría adyacente, etc.). Además, fue necesario contar con un número elevado de probetas o especímenes, que tuvieran cierta trazabilidad, y que representaran no sólo valores habituales de rugosidad, sino también asociados a materiales difíciles y en casos extremos de digitalizado (rugosidades muy altas o muy bajas, etc.).

Se han utilizado varios juegos completos de patrones de rugosidad, tipo "rugotest" de TESAC, clasificados según grados de rugosidad (ISO 468, 1982; ISO 2632-1,2, 1985) desde N1 hasta N10 y N11 según distintos procesos. Para comprobar los valores de rugosidad, to-dos ellos han sido medidos con un rugosímetro (rugotest T10 de TESA) obteniéndose, además de Ra, otros parámetros como Rq, Rt, Rz, Rsm, etc., así como las gráficas de los perfiles (Real, Filtrado y Ondulación) por si fueran necesarios para su posterior estudio y contraste. La razón de esto último es que el grado $\mathrm{N}$ de rugosidad ISO es en realidad un rango de valores del parámetro de rugosidad Ra (media aritmética).

Desde el punto de vista del procedimiento de ensayo, y además de trabajar siempre en ausencia de luz, era necesario trabajar siempre con la misma región del espacio de cada probeta para poder comparar unos ensayos con otros. Para ello se ha utilizado una placa rígida con una ranura en su interior y tres esferas de precisión pintadas en blanco mate. La placa fue pintada en negro brillante (reflectante) lo que aseguraba que el sensor nunca fuera capaz de detectarla. Las tres esferas, que sí eran detectadas por el sensor junto con la superficie de las probetas de rugosidad, permiten referenciar el digitalizado, alineando con tres puntos (centros de las esferas) en el espacio.

Sobre el sistema de coordenadas basado en estas esferas, se proyecta siempre un mismo rectángulo de control (Fig. 3) de menor tamaño que la ranura de la placa, de forma que se evita el efecto perturbador de los puntos capturados en el borde vertical de la ranura. De hecho todos los digitalizados realizados sobre probetas planas se ajustaban a un plano con buena precisión y en ensayos de repetibilidad, bajo las mismas condiciones pero alejados en el tiempo. Las dispersiones en cuanto a número de puntos y su posición siempre han sido inferiores al 0,2\%.

A la izquierda de la Fig. 3 puede observarse la placa utilizada para los ensayos colocada sobre uno

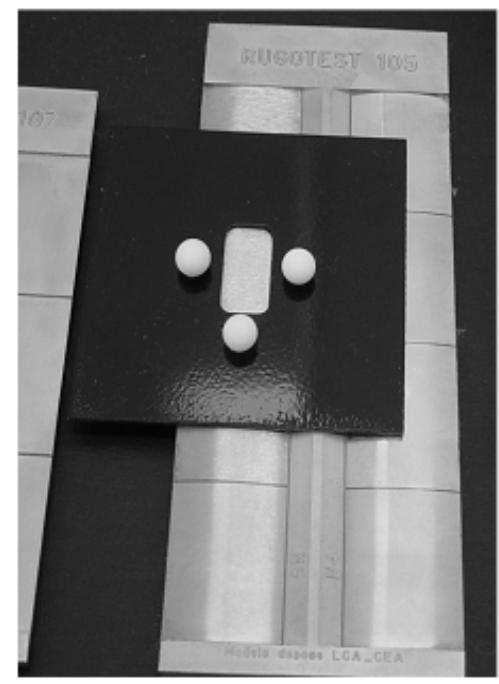

(a)

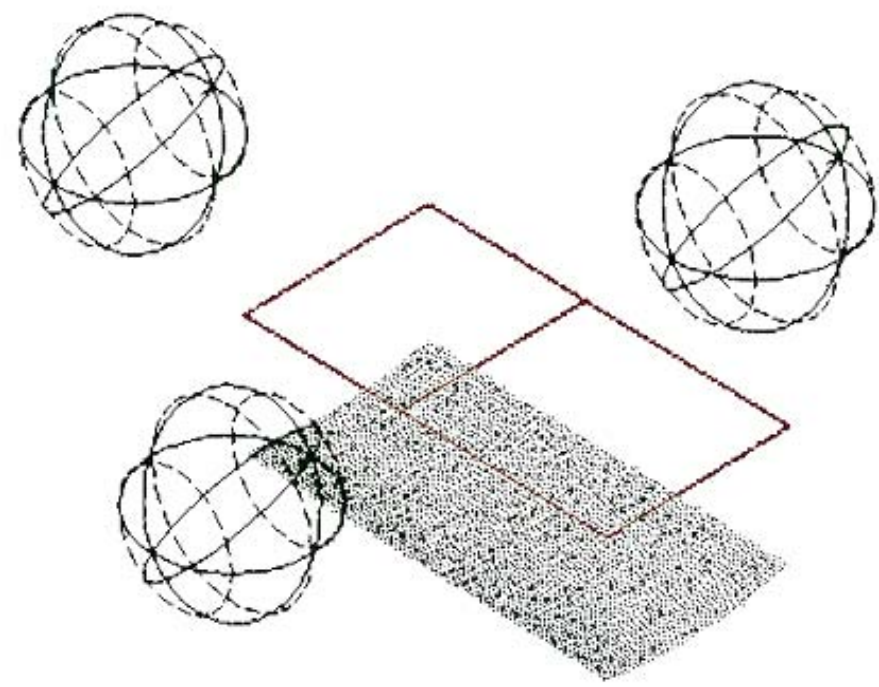

(b)

Fig. 3: (a) Placa de ensayos sobre una probeta de rugosidad. (b) Nube de puntos obtenida por referencia sobre las bolas y filtrada sobre la proyección de un rectángulo de control. 
de los patrones de rugosidad. En la parte derecha se observa una nube de puntos una vez filtrada y recortada, proyectando siempre la misma región rectangular.

La orientación de la línea de la cortina láser sobre la placa se dispuso siempre de la misma forma, de manera que se evitara el error del coseno (de desalineación) entre distintos ensayos. La alta densidad escogida para la captura de puntos (se recogieron puntos con densidad de $0.1 \times 0.1 \mathrm{~mm}$ ) también minimizaba este error a la vez que permitía que la nube fuera realmente sensible a los cambios en la rugosidad superficial, a pesar de que con ello se ralentizaron enormemente los ensayos.

En estudios preliminares (Keferstein y Marxer, 1998; Cuesta et al., 2005; Santolaria et al., 2005) se constató claramente que la variable más significativa para este estudio era, además del proceso de fabricación de cada probeta (tipo de superficie), la intensidad del láser o en su defecto, el tiempo de exposición. La densidad o tamaño de rejilla de la nube de puntos emitida no resultó significativa. Además el filtrado de saturación de puntos que se realiza por software se fijó al máximo, es decir, se admitían sólo puntos con mínima saturación o, lo que es lo mismo, con máxima calidad desde el punto de vista del CCD.

De esta forma el procedimiento de ensayo fue realizado variando la intensidad del láser, para las distintas probetas de acero (clases de rugosidad N1, N2, .., N11) correspondientes a los procesos de: electroerosión, escariado, lapeado, torneado, cepillado, fresado horizontal (periférico), fresado vertical (frontal), granallado angular, granallado esférico, limado en cruz, limado longitudinal, rectificado a mano, rectificado cilíndrico y rectificado plano. También se realizaron pruebas sobre probetas procedentes de tres procesos de conformado plástico de chapa: embutición, extrusión y laminación.

El objetivo último de los ensayos es analizar al menos dos factores: Por un lado, la variación en el número máximo de puntos capturados por el sensor al ir modificando la intensidad y, por otro, la calidad de dicha captura, entendida ésta por la forma o distribución de los puntos a la hora de construir el plano de la probeta (distribución de la planicidad). Ambos efectos se estudiaron para los distintos acabados superficiales de los distintos procesos.

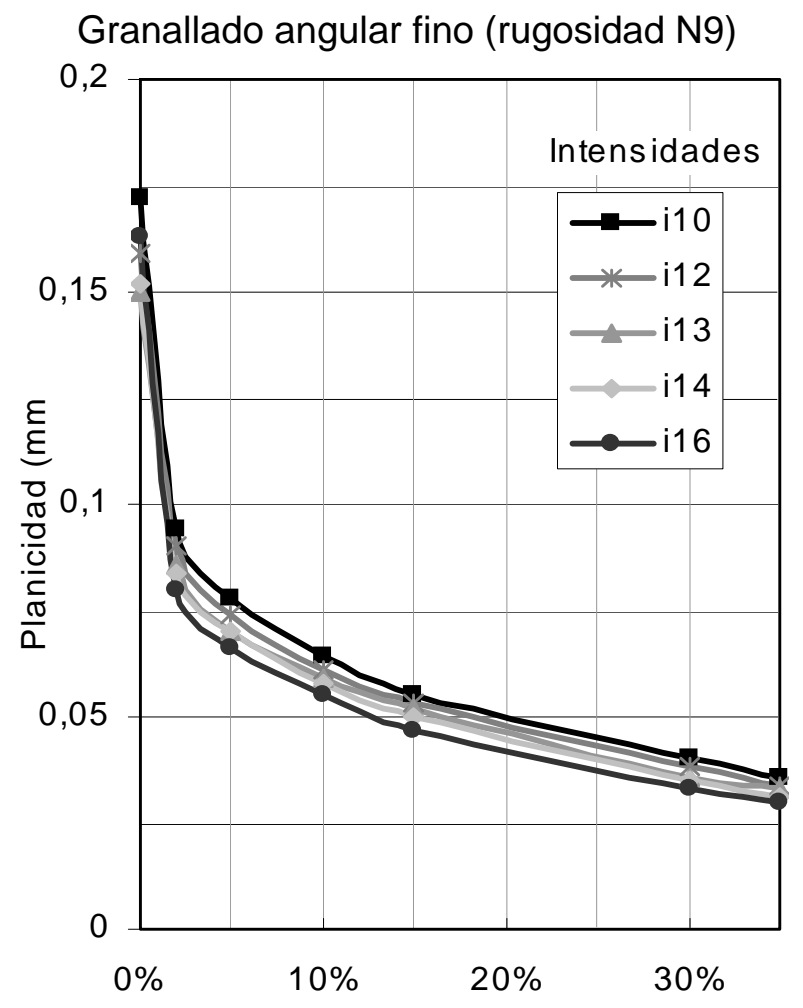

$\%$ Filtro de puntos mas alejados

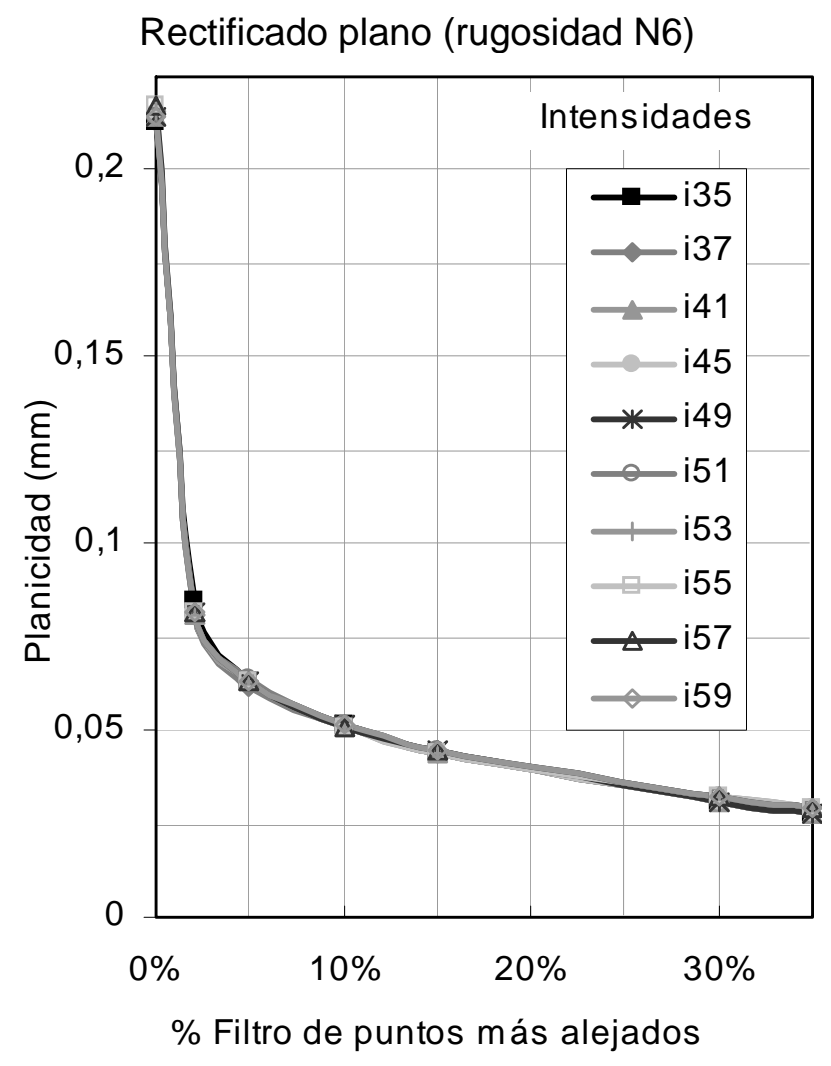

$\%$ Filtro de puntos más alejados

Fig. 4: Ejemplos de distribuciones de los puntos sobre probetas de rugosidad. 


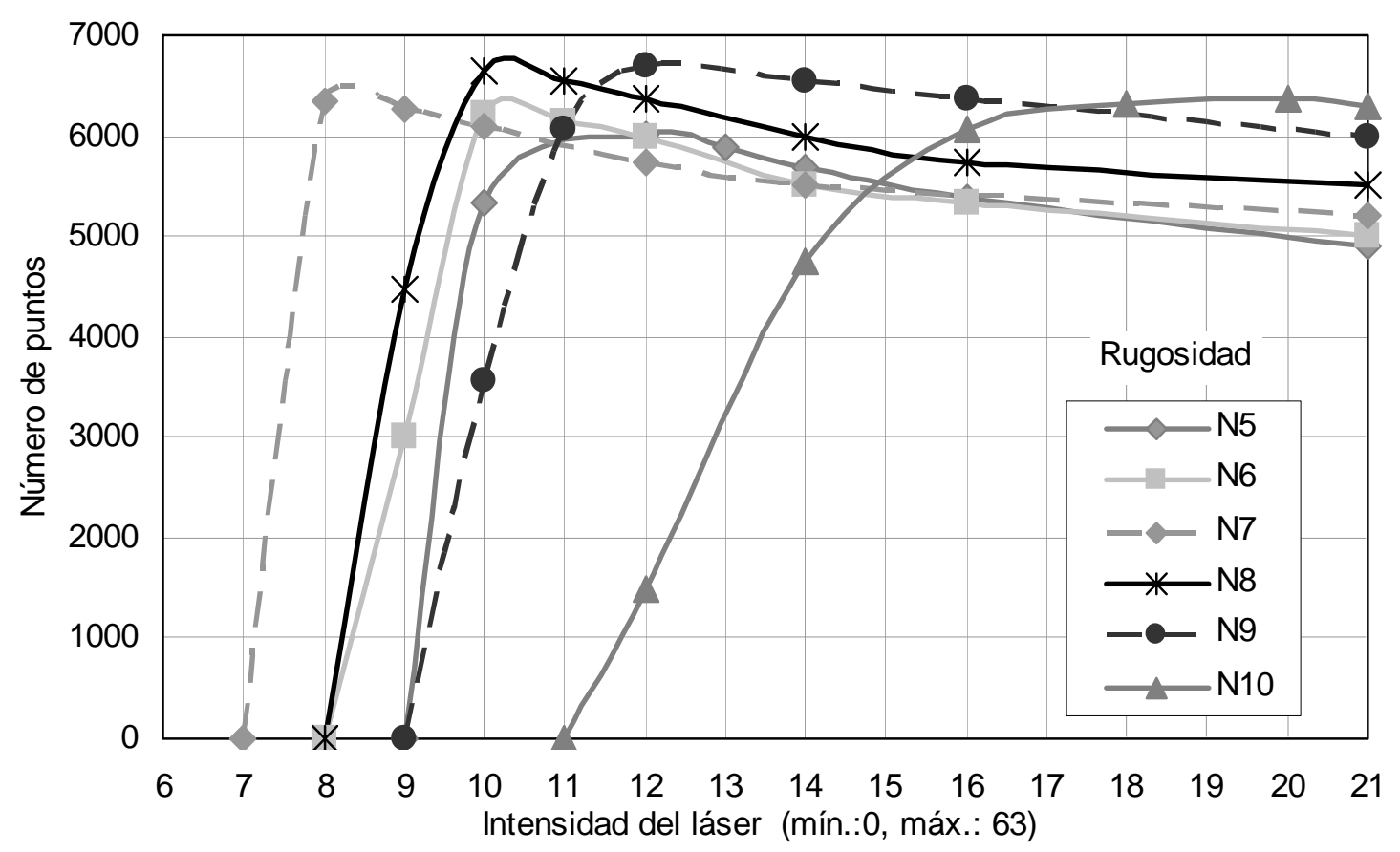

Fig. 5: Calidad del digitalizado en electroerosión.

\section{RESULTADOS Y DISCUSIÓN}

El resultado de los ensayos se materializó en una serie completa de gráficos relativos a la calidad del digitalizado (Figuras 4, 5 y 6). El estudio de la dispersión de los puntos perpendicular al plano de la probeta patrón da una idea de la calidad del digitalizado en base a la planicidad obtenida en cada ensayo y al porcentaje de puntos responsable de ese valor máximo de planicidad (distribución). Por ejemplo, en la Fig. 4 se puede comprobar que dentro de la nube de puntos capturada por el sensor, entre un $2 \%$ y un $5 \%$ de los puntos son responsables del casi el $60 \%$ del valor la planicidad. Es decir, hay una serie pequeña de puntos, distribuidos aleatoriamente, que se alejan excesivamente del plano medio y que son los causantes de una pérdida muy importante de precisión. Eliminando estos puntos la calidad del digitalizado se duplica (el error de planicidad se reduce casi a la mitad) prácticamente en casi todos los procesos con probetas planas.

Otro grupo de gráficos obtenidos fueron los que relacionaban la intensidad con el número de puntos (Fig. 5). Estos permitieron comprobar que en cada proceso existe una zona de intensidad óptima, correspondiente a la máxima captura de puntos, bastante bien definida en sentido creciente. Pero a medida que se alcanza esa zona y se sobrepasa el valor óptimo de intensidad, el decremento en el número de puntos no es tan significativo.

Un fenómeno que también se ha puesto de manifiesto y que no era predecible inicialmente ha sido la variación que se producía en la posición de la nube de puntos al variar la intensidad. En este sentido se comprobó que en la mayoría de los procesos, al aumentar la intensidad, la altura (Z) del centro geométrico de la nube de puntos disminuía, es decir, con mayor intensidad la nube capturada se situaba por debajo de la anterior. Esto ha dado lugar a otro conjunto de gráficos, similares a los de la Fig. 6, en los que se detalla el desplazamiento en la posición central del plano capturado.

El amplio número de experimentos llevados a cabo ha permitido realizar una comparativa entre los distintos procesos, constatando comportamientos similares entre la mayoría de ellos, pero resaltando algún caso anómalo como el del rectificado, que en sus distintas variantes (cilíndrico, plano y manual) se comporta ópticamente de forma distinta, y en algunos casos inversa, en cuanto a la variación de la altura de la nube de puntos. El digitalizado por triangulación con este tipo de sensores para superficies rectificadas, con alta reflectividad, no ofrece garantías, pues la zona óptima de máxima captura de puntos se produce a intensidades muy altas, tanto más altas cuanto menor sea la 


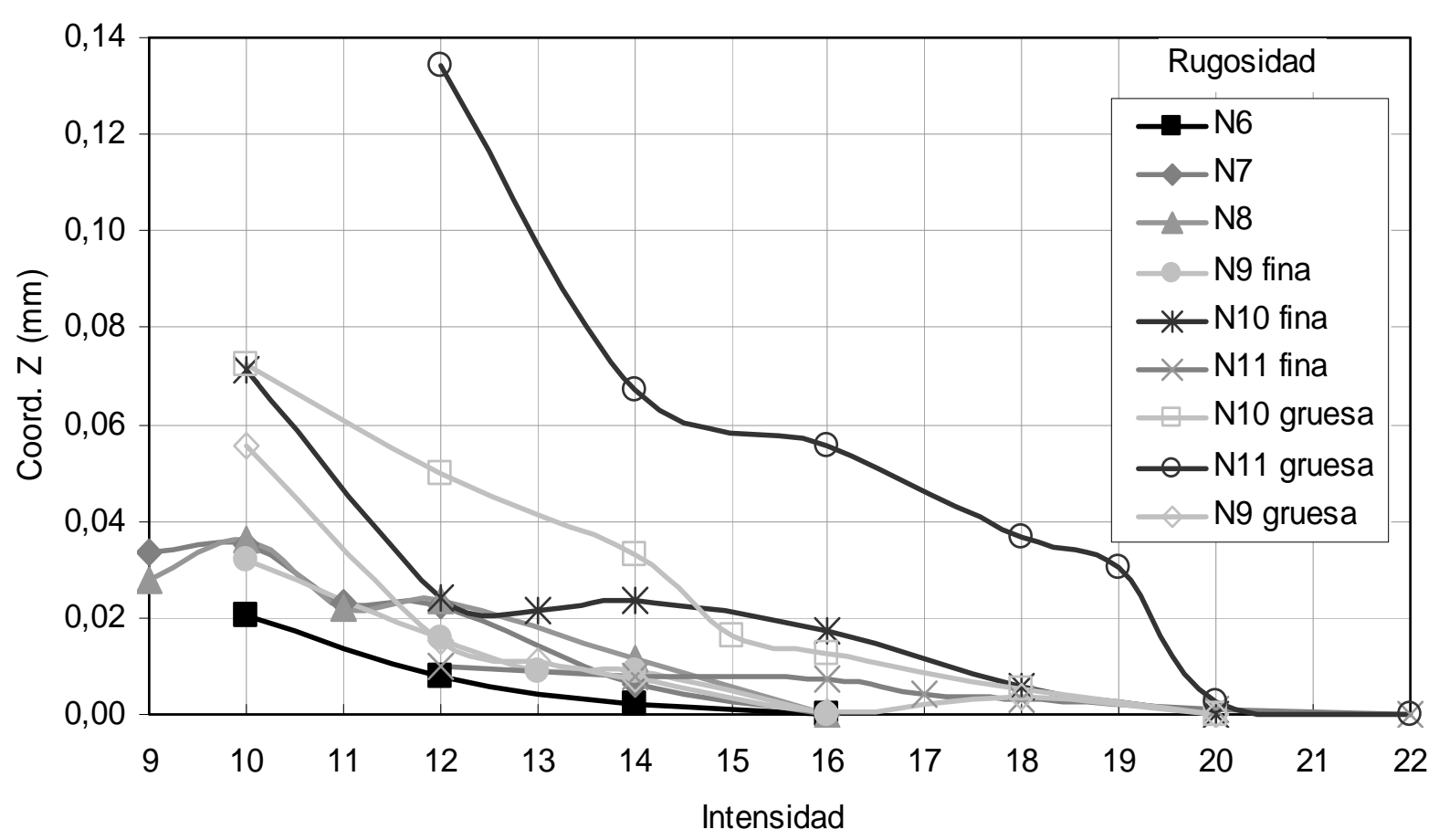

Fig. 6: Variación de la posición (Z) de la nube de puntos con la intensidad en granallado angular.

rugosidad. Incluso en esa zona el número de puntos y la calidad de la nubes son muy pobres en comparación con el resto de procesos.

\section{CONCLUSIONES}

En cuanto a la relación entre el número de puntos y la intensidad, queda claro que existe una zona bien definida de "intensidad óptima" de captura para cada proceso. En general, dicha zona se modifica en sentido creciente al desplazarnos a rangos de rugosidades mayores dentro del mismo proceso. Se aconseja por tanto digitalizar con cierta saturación (pequeño exceso de intensidad) que siempre permitirá una mayor captura de puntos a costa de una pérdida aceptablemente pequeña de precisión.

El valor total de la planicidad cuando se consideran todos los puntos de la nube, e independientemente de la distribución de los puntos dentro de la nube, ha sido en el mejor de los casos muy superior al encontrado utilizando palpado por contacto. Sin embargo, la forma de la distribución (Fig. 4) se corresponde con una exponencial de forma que eliminando tan solo el $2 \%$ de los puntos más alejados la planicidad se reduce a más de la mitad en la mayoría de los casos. Esto apunta claramente a una mejora factible en los programas de captura, software de control del sensor, por parte de los fabricantes de estos equipos. La forma de esta distribución es atribuible en principio a la alta reflexión de las superficies metálicas utilizadas, pero permite extrapolar la forma de la curva cuando se digitalicen otros tipos de superficies oscuras (materiales plásticos, etc.).

La variación de la posición de la nube de puntos con la intensidad aconseja digitalizar siempre con la misma intensidad. En caso de problemas derivados de nubes de puntos incompletas, falta de definición, etc., es preferible variar la posición del sensor (por ejemplo, jugando con la programación de trayectorias) para capturar nuevos puntos, en vez de incrementar la intensidad. Esto último daría lugar a una nube de puntos más densa, pero con un error en su posición (desplazamiento).

Se ha comprobado que existen procesos de fabricación que presentan acabados superficiales con propiedades ópticas óptimas de difusión de luz para el digitalizado con este tipo de sensores de triangulación (p.e. electroerosión), a pesar de tener rugosidades elevadas, lo que sin embargo los invalidan como patrones para palpado por contacto.

Por último, señalar que las conclusiones son extrapolables a otros sistemas de este tipo que utilicen 
la variable tiempo de exposición en vez de intensidad en cuanto a tendencia, pero no en cuanto a magnitud de las diferencias encontradas, puesto que esto dependerá de las características constructivas del sensor en particular, algoritmos de calibración, etc.

\section{AGRADECIMIENTOS}

Este trabajo forma parte de los resultados obtenidos en el marco de un Proyecto de Investigación financiado por el Ministerio de Educación y Ciencia de España (Ref. DPI2004-03517) y FEDER.

\section{REFERENCIAS}

Cuesta, E. y otros cuatro autores; Aplicaciones metrológicas y de ingeniería inversa de los láseres por triangulación, Deformación Metálica: 284, 59-73 (2005).

Curless, B. y M. Levoy; Better optical triangulation through spacetime analysis, IEEE International Conference on Computer Vision, 5, 987-994, Boston, USA, 23 al 25 de Junio (1995).

Feng H-Y., Y. Liu y F. Xi; Analysis of digitizing errors of a laser scanning system, Precision Engineering: 25(3), 185-191 (2001).

Keferstein, C.P. y M. Marxer; Testing bench for laser triangulation sensors, Sensor Review: 18(3), 183-187 (1998).

Keferstein, C.P. y R. Zust; Minimizing technical and financial risk when integrating and applying optical sensors for in-process measurement, International Intelligent Manufacturing Systems Forum, Cernobbio, Italia, 17 al 19 de Mayo (2004).

ISO 2632-1,2; Roughness comparison specimens, 1-6 y 1-5, Ginebra, Suiza (1985).

ISO 468; Surface roughness - Parameters, their values and general rules for specifying requirements, 1-6, Ginebra, Suiza (1982).

Lai, X., Z. Lin, T. Huang y Z. Zeng; A study of a reverse engineering system based on vision sensor for free-form surfaces, Computers and Industrial Engineering: 40(3), 215-227 (2001).

$\mathrm{Li}$, L. y otros cuatro autores; A reverse engineering system for rapid manufacturing of complex objects, Robotics and Computer-Integrated Manufacturing: 18(1), 53-67 (2002).

Rico, J.C. y otros cuatro autores; Evaluación de los parámetros de influencia en el digitalizado por láser de barrido sobre una MMC, Congreso Español de Metrología (CEM), 3, Zaragoza, España, 11 al 13 de Mayo (2005a).

Rico, J.C. y otros cuatro autores; Establecimiento de una metodología de trabajo para el digitalizado mediante un láser de barrido sobre $M M C$, Congreso Internacional de la Sociedad de Ingeniería de Fabricación, 1, Calatayud, España, 19 a 21 de Septiembre (2005)

Santolaria, J., J. Puertas, M.A. Lope y J.J. Aguilar; Análisis de precisión de Sensores Láser por Triangulación: Aplicación a la digitalización de Superficies, Congreso Internacional de la Sociedad de Ingeniería de Fabricación, 1, Calatayud, España, 19 a 21 de Septiembre (2005)

Son, S., H. Park y K.H. Lee; Automated laser scanning system for reverse engineering and inspection, International Journal of Machine Tools \& Manufacture: 42(8), 889-897 (2002).

Xi, F. y C. Shu; CAD-based path planning for 3-D line laser scanning, Computer Aided Design: 31(7), 473-479 (1999). 